\title{
Perfil clínico y costos directos de la atención de asma en una red de instituciones de salud en Bogotá
}

Diego Rosselli ${ }^{1}$, Yaneth Gil ${ }^{2}$, Pieralessandro Lasalvia ${ }^{2}$, Maria Paula Peña ${ }^{2}$, Camilo Castañeda ${ }^{2}$, Óscar Virgüez ${ }^{3}$, Andrés González ${ }^{4}$, Carmen García ${ }^{4}$, María Laucho-Contreras (1) Pontificia Universidad Javeriana, (2) Neuroeconomix, (3) Virrey Solis IPS, (4) GlaxoSmithKline Colombia

Introducción

El asma es la más prevalente de las enfermedades respiratorias crónicas en Colombia, implicando una gran carga clínica y económica al sistema de salud (1).

Objetivo

Describir las caracteristicas clínicas, uso de recursos y costos directos de la atención del asma en una red de instituciones prestadoras en salud de Bogotá.

Métodos

- Diseño: Estudio descriptivo con recolección retrospectiva de datos.

- Población: Pacientes con diagnóstico de asma atendidos en Virrey Solis IPS (red de atención primaria y urgencias) entre octubre de 2014 y marzo de 2017 con al meno una consulta y una prescripción asociados a la enfermedad. Se excluyeron pacientes con comorbilidad respiratoria significativa incluyendo EPOC.

- Información clínica: Los datos clínicos y de costos del año de observación fueron extraídos de las historias clínicas electrónicas y sistemas de información administrativos. La gravedad de la enfermedad se determinó a partir de los pasos de GINA 2017.

- Costos: Para el cálculo del costo directo se multiplicó la frecuencia medida de uso de recursos por los precios 2018 obtenidos de manuales tarifarios SOAT e ISS y la base SISMED 2018 según metodología IETS (2). Una estimación teórica independiente del uso de recursos realizada por cuatro neumólogos fue costeada y cotejada con los hallazgos observados.

\section{Resultados}

$\checkmark 7919$ pacientes incluidos, $86 \%$ con asma leve o moderada (tablas 1 y 2 ).

$\checkmark$ El costo directo anual promedio de la atención de asma leve, moderada y grave según tarifario SOAT fue de $502.752,769.454$ y 5.924.398 pesos respectivamente.

$\checkmark$ El costo total observado fue inferior al estimado por los expertos (tabla

$\checkmark$ Los medicamentos representaron el $79 \%$ de los costos totales.

$\checkmark$ Las hospitalizaciones, aunque poco frecuentes, fueron de alto costo (mediana por episodio \$976.401).

\section{Conclusiones}

El costo promedio de atención del asma es altamente variable, siendo en

su mayoría causado por el costo de los medicamentos. Se evidencia una

tendencia entre el costo y la gravedad de la enfermedad.

Punto de discusión

La baja frecuencia y costo promedio de las hospitalizaciones podría ser explicada por la distribución de la gravedad del asma y por el uso de medicamentos de control ( $63 \%$ corticosteroide inhalado)

\begin{tabular}{|c|c|c|c|c|c|c|c|c|c|c|c|c|}
\hline \multicolumn{3}{|c|}{ Tabla 1: Características clinicas } & \multicolumn{5}{|c|}{ Tabla 2: Frecuencia individual de desenlaces y uso de recursos } & \multicolumn{5}{|c|}{ Tabla 3: Costos directos de la atención de asma, en miles de pesos } \\
\hline & $n$ & $\%$ & & Minimo & Mediana & Promedio & Máximo & & Minimo & Mediana & Promedio & Máximo \\
\hline \multicolumn{3}{|l|}{ Grupo de edad } & Eventos relacionados con el asma & & & & & Costo anual por persona & & & & \\
\hline Infante $(<12$ años) & 3.707 & 46,81 & Exacerbaciones ${ }^{*}$ & 0.0 & 10 & 127 & 250. & Todos los pacientes (ISS+30\%) & 0 & 111 & 561 & 197.502 \\
\hline Adolescente (12 a 18 años) & 778 & 9,82 & Consultas ambulatorias & 0,0 & 4,0 & 4,49 & 41,0 & $\begin{array}{l}\text { Todos los pacientes (SOAT) } \\
\text { (SO }\end{array}$ & 0 & 232 & 711 & 197.768 \\
\hline Adulto (19 a 65 años) & 3.201 & $\begin{array}{r}3,002 \\
40,42\end{array}$ & Visitas a urgencias & 0,0 & $\begin{array}{l}1,0 \\
1,0\end{array}$ & $\begin{array}{l}4,15 \\
1,15\end{array}$ & 24,0 & Adultos clasificables" (ISS $+30 \%$ ) & 0 & $\begin{array}{r}202 \\
99\end{array}$ & 625 & 197.502 \\
\hline Adulto mayor (>65 años) & 233 & 2,94 & Espirometrias & 0,0 & 0,0 & 0,09 & 3,0 & Estimado por expertos & 998 & 1.451 & 1.455 & 2.166 \\
\hline Sexo femenino & 4419 & $\begin{array}{r}2,34 \\
55,73\end{array}$ & Hospitalizaciones & 0,0 & 0,0 & 0,01 & 2,0 & Adultos clasificables (SOAT) & 3 & 207 & & 197.768 \\
\hline \multicolumn{3}{|l|}{$\begin{array}{l}\text { Sexotemenino } \\
\text { Raza }\end{array}$} & & & & & & Estimado por expertos & 1.638 & 2.073 & 2.181 & 3.288 \\
\hline Mestiza & 3.337 & 42,14 & & & $n$ & & & & & & & \\
\hline Blanca & 626 & 7,91 & Una o más exacerbaciones & & 4.388 & & 55,4 & Costo según categoria (todos los & & & & \\
\hline Negra & 105 & 1,33 & Una o más consultas a médico & & & & & $\begin{array}{l}\text { pacientes) } \\
\text { (n) }\end{array}$ & & & & \\
\hline Indigena & 15 & 0,19 & especialista & & 3.772 & & 47,6 & Atención ambulatoria (ISS+30\%) & 0 & 82 & 112 & 13.388 \\
\hline Sin dato & 3.836 & 48,44 & Una o más visitas a urgencias & & 4.008 & & 50,6 & Atención ambulatoria (SOAT) & 0 & 202 & 262 & 3.631 \\
\hline \multicolumn{3}{|l|}{$\begin{array}{l}\text { Sin dato } \\
\text { Estrato socloeconómico }\end{array}$} & Frecuencia estimada por expertos & & & & 60,0 & Medicamentos (SISMED 2018) & 0 & 20 & 444 & 197.460 \\
\hline $\begin{array}{l}\text { Istratá socloceconomilco } \\
1 \text { (más bajo) }\end{array}$ & 60 & 0,76 & Una o más consultas a otros & & & & & Hospitalización (datos administrativos) & 0 & 0 & 5 & 12.747 \\
\hline 2 & 11 & 0,14 & profesionales & & 2.464 & & 31,1 & & & & & \\
\hline 3 & 7.146 & 90,24 & Una o más hospitalizaciones & & 54 & & 0,7 & Costo según categoria (adultos & & & & \\
\hline 4 & & $\begin{array}{r}90,24 \\
0,05\end{array}$ & Frecuencia estimada por expertos & & & & 40,0 & clasificables) & & & & \\
\hline $\begin{array}{l}4 \\
5 \text { (más altol }\end{array}$ & ${ }_{1}^{4}$ & 0,05 & Cuidados intermedios o intensivos & & 12 & & 0,2 & Atención ambulatoria (ISS $+30 \%$ ) & 0 & 70 & 105 & 4.274 \\
\hline $\begin{array}{l}5 \text { (más atto) } \\
\text { Sin dato }\end{array}$ & 1 & 0,01 & Frecuencia estimada por expertos & & & & 2,0 & Atención ambulatoria (SOAT) & 0 & 177 & 241 & 2.238 \\
\hline \multicolumn{3}{|l|}{$\begin{array}{l}\text { Sin dato } \\
\text { Gravedad del asma". }\end{array}$} & Medicamentos & & & & & Medicamentos (SISMED 2018) & 2 & 21 & 514 & $\begin{array}{ll}2.250 \\
197.460\end{array}$ \\
\hline & & & SABA & & 7.031 & & 88,8 & Hospitalización (datos administrativos) & 0 & 0 & 8 & 12.747 \\
\hline Leve & 3.154 & 39,83 & Antihistamínicos & & 5.368 & & 67,8 & & & & & \\
\hline Moderado & 3.652 & 46,12 & Corticosteroides inhalados & & 5.011 & & 63,3 & Costo según gravedad (ISS+30\%, todos) & & & & \\
\hline Grave & 70 & 0,88 & $\begin{array}{l}\text { Corticosteroides orales } \\
\text {. }\end{array}$ & & 2.180 & & 27,5 & Leve & 3 & 99 & 364 & 4197.502 \\
\hline \multirow{2}{*}{\multicolumn{3}{|c|}{$\begin{array}{l}\text { No clasificable } \\
\text { Comorbilidades presentes }\end{array}$}} & SAMA & & 1.265 & & $\begin{array}{l}27,0 \\
16,0\end{array}$ & Moderada & 5 & 122 & 503 & 197.768 \\
\hline & & & SAlum & & $\begin{array}{r}.6265 \\
652\end{array}$ & & $\begin{array}{r}16,0 \\
82\end{array}$ & $\begin{array}{l}\text { Woueradad } \\
\text { Grave }\end{array}$ & 71 & 1.173 & 5.712 & 90.944 \\
\hline Rinitis alérgica & 1.631 & 20,60 & $\begin{array}{l}\text { Comogicato } \\
\text { Antileucotrienos }\end{array}$ & & $\begin{array}{l}652 \\
580\end{array}$ & & $\begin{array}{l}8,2 \\
7,3\end{array}$ & No clasificable & 0 & 100 & $\begin{array}{r}632 \\
632\end{array}$ & 135.811 \\
\hline Eczema & 669 & 8,45 & ICS/LABA & & 345 & & $\begin{array}{l}4,3 \\
4,4\end{array}$ & No clasificable & & & & \\
\hline Obesidad & 664 & 8,38 & Xantinas & & 72 & & 0,9 & Costo según gravedad (estimación & & & & \\
\hline Conjuntivitis & 406 & 5,13 & SABA/SAMA & & 19 & & 0,2 & expertos) & & & & \\
\hline $\begin{array}{l}\text { Rinitis crónica } \\
\text { lata }\end{array}$ & 307 & 3,88 & Omalizumab & & 17 & & $\begin{array}{l}0,2 \\
0,2\end{array}$ & $\begin{array}{l}\text { expertis) } \\
\text { Leve }\end{array}$ & 292 & 517 & 548 & 866 \\
\hline \multirow{3}{*}{$\begin{array}{l}\text { Factores de riesgo } \\
\text { Tabaquismo (12+ años) } \\
\text { Exposición pasiva a } \\
\text { cigarrillo }\end{array}$} & \multirow[b]{2}{*}{80} & \multirow[b]{2}{*}{1,90} & Mucoliticos & & $\begin{array}{r}11 \\
4\end{array}$ & & $\begin{array}{l}0,2 \\
0,1\end{array}$ & $\begin{array}{l}\text { Leve } \\
\text { Moderada }\end{array}$ & $\begin{array}{r}292 \\
1.663\end{array}$ & 2.533 & $\begin{array}{r}548 \\
2.499\end{array}$ & $\begin{array}{r}866 \\
3.266\end{array}$ \\
\hline & & & \multirow{3}{*}{\multicolumn{5}{|c|}{$\begin{array}{l}\text { SABA: beta agonista de acción corta; SAMA: antagonista muscarinico de } \\
\text { acción corta; ICS/LABA: combinación de corticosteroide inhalado y beta }\end{array}$}} & & $\begin{array}{l}.1 .030 \\
3.301\end{array}$ & 6.340 & $\begin{array}{l}.2499 \\
6.543\end{array}$ & $\begin{array}{r}3.200 \\
10.193\end{array}$ \\
\hline & 131 & 1,65 & & & & & & Costo según grupo de edad (ISS+30\%) & & & & \\
\hline \multirow{5}{*}{\multicolumn{3}{|c|}{$\begin{array}{l}\text { * Clasificada según pasos GINA 2017: asma leve } \\
\text { (pasos } 1 \text { y 2), moderada (paso 3) y grave (pasos } 4 \\
\text { y } 5 \text { ) } \\
\text { † El tratamiento recibido no coincide con ningún } \\
\text { paso de GINA } \\
\text { t Se muestran las cinco comorbilidades más } \\
\text { frecuentes }\end{array}$}} & & & & & & Infante (<12 años) & 0 & 124 & & 117.131 \\
\hline & & & \multirow{4}{*}{\multicolumn{5}{|c|}{$\begin{array}{l}\text { agonista de acción larga. } \\
\text { * Una exacerbación del asma se definió como: 1) consulta de urgencias } \\
\text { relacionada con asma o admisión hospitalaria relacionada con asma, o 2) uso } \\
\text { de corticosteroides orales } \geq 20 \mathrm{mg} \text { prednisona o equivalente por al menos } 3 \\
\text { dias (pero no más de } 28 \text { dias) con un registro diagnóstico de asma dentro de } \pm \\
2 \text { semanas. }\end{array}$}} & Adolescente (12 a 18 años) & 0 & 69 & 218 & 57.475 \\
\hline & & & & & & & & Adulto ( 19 a 65 años) & 0 & 105 & 632 & 197.502 \\
\hline & & & & & & & & Adulto mayor (>65 años) & 11 & 190 & 2.657 & 135.299 \\
\hline & & & & & & & & $\begin{array}{l}\text { Valores en miles de pesos. Las cifras estáa } \\
{ }^{*} \text { Mayores de } 12 \text { años con clasificación de }\end{array}$ & $\begin{array}{l}\text { redondeac } \\
\text { gravedad d }\end{array}$ & $\begin{array}{l}\text { adas hacia a } \\
\text { de la enfern }\end{array}$ & $\begin{array}{l}\text { arriba. } \\
\text { medad }(n=3\end{array}$ & \\
\hline
\end{tabular}

Declaraciones: Este estudio fue patrocinado por GSK, código HO-16-16570. AG, CG y MELC son empleados y/o accionistas de GSK. YG, PL, MPP, CC y DR recibieron honorarios por el proyecto de investigación, pero su actuar científico fue independiente al patrocinio.

Referencias

1. Ministerio de Salud y Protección Social, Organización Panamericana de la Salud. Morbi-mortalidad de las enfermedades respiratorias crónicas, Colombia 2005-2013 2016.

2. Instituto de Evaluación Tecnológica en Salud - IETS. Manual metodológico para la elaboración de evaluaciones de efectividad, seguridad y validez diagnóstica de tecnologias en salud. Bogotá D.C. 2014 